\title{
WHAT CAN WE LEARN FROM SEMI-INCLUSIVE RAPIDITY CORRELATIONS?
}

\author{
F. HAYOT * and M LE BELLAC ** \\ Department of Physics, Unversity of Michigan ${ }^{\dagger}$
}

Received 24 May 1974

(Revised 22 July 1974)

\begin{abstract}
We study a general formulation of semi-inclusive two-particle rapidity correlations for short-range models We use it to compare with the $205 \mathrm{GeV}$ NAL Bubble Chamber data different decay distributions for independently emitted clusters We also comment on non-independent cluster production and on semi-1nclusive correlations between charged and neutral particles
\end{abstract}

\section{Introduction}

In this work we attempt to answer the following question. what kind of information can be obtained from the study of semi-inclusive rapidity correlations $[1,2]$ ? A first advantage of semi-inclusive correlations has already been pointed out by Berger [1] if one restricts oneself to multiplicities larger than $\sim \frac{1}{2} \bar{n}$, it is possible to ignore diffractive effects (in a two-component type of model) This is certainly a very interesting point since the way to handle diffraction is far from clear We will therefore concentrate in this work on the non-diffractive component of the production mechanism

Our first goal is to derive a general formula for semi-1nclusive correlations, valid both in multiperıpheral (MP) and in cluster type models By cluster models we mean, as is now fashionable [3], production of independent neutral clusters which decay into the observed particles In sect 2 we derive in a simple and physically transparent way a formula first obtained by Arnold and Thomas [4], which allows us to calculate semi-inclusive correlations from inclusive ones. We demonstrate explicitly the equivalence between MP and cluster models which has been recently questioned [5]

In sect 3 we perform a phenomenological analysis of NAL [6] and ISR [7] data, using simple versions of the cluster model. In particular we compare various distributions of charged particles within the clusters, and obtain good agreement with the

\footnotetext{
* Permanent address Service de Physique Théorique CEN Saclay

** Permanent address Faculté des Sciences, Université de Nice

$\dagger$ Work supported by the US A tomic Energy Commission
} 
NAL data, and also with the $62 \mathrm{GeV}$ data of the P1sa-Stony Brook experiment

In sect 4 we comment about neutral charged correlations from the point of view of independent cluster models Finally, in sect 5, we look at the case of nonindependent clusters This section is rather technical. However, we point out the important fact that the combined knowledge of topological cross sections and semi-inclusive correlations gives a clue as to the cluster multiplicity distribution Our most important theoretical and phenomenological points are summarized in sect 6

\section{Semi-inclusive correlations from inclusive ones}

Let us first derive a general formula for semi-inclusive correlations, which depends only on the short range nature of the correlations, namely on the fact that the width of the multiplicity distribution $P(n)$ is of order $(n)^{\frac{1}{2}}$. We define as usual the semi-1nclusive and inclusive one and two particle distributions

$$
\begin{aligned}
& \rho_{n}(y)=\frac{1}{\sigma_{n}} \frac{\mathrm{d} \sigma_{n}}{\mathrm{~d} y}, \quad \rho(y)=\frac{1}{\sigma} \frac{\mathrm{d} \sigma}{\mathrm{d} y}, \\
& C_{n}\left(y_{1}, y_{2}\right)=\frac{1}{\sigma_{n}} \frac{\mathrm{d}^{2} \sigma_{n}}{\mathrm{~d} y_{1} \mathrm{~d} y_{2}}-\rho_{n}\left(y_{1}\right) \rho_{n}\left(y_{2}\right), \\
& C\left(y_{1}, y_{2}\right)=\frac{1}{\sigma} \frac{\mathrm{d}^{2} \sigma}{\mathrm{d} y_{1} \mathrm{~d} y_{2}}-\rho\left(y_{1}\right) \rho\left(y_{2}\right)
\end{aligned}
$$

From the obvious relation

$$
\frac{\mathrm{d}^{2} \sigma}{\mathrm{d} y_{1} \mathrm{~d} y_{2}}=\sum_{n} \frac{\mathrm{d}^{2} \sigma_{n}}{\mathrm{~d} y_{1} \mathrm{~d} y_{2}},
$$

we derive the following general expression for $C\left(y_{1}, y_{2}\right)$

$$
C\left(y_{1}, y_{2}\right)=\sum_{n} P(n) C_{n}\left(y_{1}, y_{2}\right)+\sum_{n} P(n)\left(\rho_{n}\left(y_{1}\right)-\rho\left(y_{1}\right)\right)\left(\rho_{n}\left(y_{2}\right)-\rho\left(y_{2}\right)\right)
$$

At ultra-high energy, only the first term in (1) will survive, since the second term is of the order of $1 / \sqrt{n}$, as we explain in more detall below Moreover, since the correlations are assumed to be short range, we can replace

$$
\sum P(n) C_{n}
$$

by $C_{\bar{n}}$, with an error of the order of $1 / \bar{n}$, so that

$$
C\left(y_{1}, y_{2}\right)=C_{\bar{n}}\left(y_{1}, y_{2}\right)+\mathrm{O}(1 / \bar{n}) \text {. }
$$

In order to generalize this result to arbitrary values of $n$, we modify the multiplicity distribution in the following way 


$$
P(n) \rightarrow P_{z}(n)=\frac{z^{n} P(n)}{\sum z^{n} P(n)}
$$

In the case of the MP model, $z$ is to be interpreted as the square of the coupling constant and by convention the physical value corresponds to $z=1$ For cluster models eq. (3) has only a formal meaning, but in each case we can define $z$-independent one and two-particle densities, as well as $z$-dependent moments $\bar{n}(z)$ and $f_{2}(z)$ If we choose $z$ in such a way that $n$ is precisely equal to $\bar{n}(z)^{*}$, we can generalize (2a) to

$$
C\left(y_{1}, y_{2}, z\right)=C_{n}\left(y_{1}, y_{2}, n=\bar{n}(z)\right)+\mathrm{O}(1 / n)
$$

We have checked numerically, for example in the case of cluster models with $\rho-\rho$ clusters (see sect 3 ), that the $O(1 / n)$ error in (2b) is typically of a few percent and never exceeds $15 \%$ even for low values of $n$ and low energies In the case of the cluster model considered in ref. [1] our result is even exact.

At finite (NAL and ISR) energies, we have to take into account the second term in eq (1) If we assume that $\rho(y)$ and $\rho_{n}(y)$ have each a plateau of length $Y$

$$
\rho(y)=\frac{\bar{n}}{Y}, \quad \rho_{n}(y)=\frac{n}{Y},
$$

the second term in (1) is found to be equal to $\left(n+f_{2}\right) / Y^{2}$, where $f_{2}$ is the second Mueller's moment Generalızıng to arbitrary $z$ we finally get our basic equation (first derived by Arnold and Thomas [4])

$$
C_{n}\left(y_{1}, y_{2}, n=\bar{n}(z)\right)=C\left(y_{1}, y_{2}, z\right)-\frac{1}{Y^{2}}\left(\bar{n}(z)+f_{2}(z)\right)
$$

Before turning to specific applications let us make a few comments about (4)

(1) One sees from eq (4) that the semi-inclusive correlation is given by a function depending on $y_{1}-y_{2}$ sitting on a constant negative background. This background is of the order of $1 / Y$ and thus disappears at ultra-high energy, but is quantitatively important at present energies Notice that the background term is essential in order for the sum rule

$$
\int \mathrm{d} y_{1} \mathrm{~d} y_{2} C_{n}\left(y_{1}, y_{2}\right)=-n
$$

to be satisfied In fact integratıng (4) over $\left(y_{1}, y_{2}\right)$ gives for the right-hand side $f_{2}(z)-\left(\bar{n}(z)+f_{2}(z)\right)=-n$

(1i) Experimentally it is found that at NAL energies $C_{n}(0,0)$ is close to zero and therefore that there exists at those energies an almost complete cancellation between the two terms on the right-hand side of eq (4) Thus there is some doubt whether terms of order $1 / Y$ in $C_{n}$ can be neglected as argued above before point (1) This seems a difficulty unavoidable in all short-range order approaches to semi-1nclusive correla-

\footnotetext{
* In the steepest descent method used by Arnold and Thomas [4] to derive eq (4), the value of $z$ such that $\bar{n}(z)=n$ corresponds to the position of the saddle point
} 
tions at NAL and ISR energies. Indeed, as emphasized in the following section, it would be very important to separate experimentally the two terms in (4) which differ by their $y_{1}-y_{2}$ dependence, in order to extract valuable information from the data

(i11) Eq. (4) applies to any short range correlation model The MP model does nat specify the form of the functions $\bar{n}(z)$ and $C\left(y_{1}, y_{2}, z\right)$. The cluster model is slightly more restrictive first the correlation length is $z$-independent and second, as is clear from sect. $3,\left|C_{n} / n\right|$ is a non-decreasing function of $n$ This last behavior may turn out to be different in specific versions of the MP model, but there does not seem to be any fundamental difference between the MP and cluster models as far as sem1inclusive correlations are concerned, contrary to what has been asserted by Caneschi [5]

\section{Independent cluster models}

We now apply eq (4) to the cluster model. We call $w(p)$ the probability that a given cluster decays into $p$ observed (charged, or negative) particles, and $\lambda(z)$ the generating function for the cluster decay

$$
\lambda(z)=\sum_{p} z^{p} w(p)
$$

Since the clusters are assumed to be independent and neutral, the generating function for the total multiplicity distribution $G(z)$ is given by $[8,9]$

$$
G(z)=\exp \{\bar{k}(\lambda(z)-1)\},
$$

where $\bar{k}$ is the average number of clusters. We then have.

$$
\begin{aligned}
& \bar{n}(z)=\bar{k} z \lambda^{\prime}(z)=\bar{k}\langle p\rangle_{z}, \\
& f_{2}(z)=\bar{k} z^{2} \lambda^{\prime \prime}(z)=\bar{k}\langle p(p-1)\rangle_{z},
\end{aligned}
$$

and the inclusive correlation has the form [10]

$$
C\left(y_{1}, y_{2}\right)=\bar{k}\langle p(p-1)\rangle_{z} P^{(2)}\left(y_{1}, y_{2}\right) \text {, }
$$

where $P^{(2)}$ is normalized to unity In order to be definite, let us write the formulae in the case of $y_{1}=0$, assuming that the rapidity distribution in the cluster rest frame is a Gaussian of width $\delta$, with $\delta \approx 07$ Then [11]

$$
P^{(2)}(0, y)=\frac{1}{2 \delta \sqrt{\pi} Y} \mathrm{e}^{-y^{2} / 4 \delta^{2}},
$$

and we find *

* In MP models the Gaussian in $y$ should be replaced by an exponential, and the correlation length may depend on $z$ 


$$
C_{n}(0, y)=-\frac{1}{Y^{2}}\left(n+\bar{k}\langle p(p-1)\rangle_{z}\right)+\frac{\bar{k}\langle p(p-1)\rangle_{z}}{2 \delta \sqrt{\pi} Y} \mathrm{e}^{-Y^{2} / 4 \delta^{2}}
$$

Let us recall that $z$ is to be determined by the equation $\bar{k} z \lambda^{\prime}(z)=n$ In order to compare (6) with experiment, it is more convenient to work with the ratio $R_{n}$, defined as usual by

$$
R_{n}\left(y_{1}, y_{2}\right)=\frac{C_{n}\left(y_{1}, y_{2}\right)}{\rho_{n}\left(y_{1}\right) \rho_{n}\left(y_{2}\right)}
$$

Using (6) we find for $R_{n}$.

$$
R_{n}(0, y)=-\frac{1}{n}\left(1+\frac{\langle p(p-1)\rangle_{z}}{\langle p\rangle_{z}}\right)+\frac{\langle p(p-1)\rangle_{z}}{\langle p\rangle_{z}} \frac{Y}{n} \frac{\mathrm{e}^{-y^{2} / 4 \delta^{2}}}{2 \delta \sqrt{ } \pi}
$$

Eq (7) is the basic formula which we will compare with the experimental data It is valid for the class of models where neutral clusters are produced independently The particular case where each cluster decays into a fixed number of particles has been treated in ref [1] The most important point about eq (7) is that $R_{n}$ (or $C_{n} / n$ ) depends only on the ratio $\langle p(p-1)\rangle_{z} /\langle p\rangle_{z}$, and it would be extremely interesting to extract this ratio from the experimental data

As suggested by Berger [1], one can fit $R_{n}(0, y)$ with the formula [12]

$$
R_{n}(0, y)=-\frac{A_{n}}{n}+B_{n} \frac{\mathrm{e}^{-y^{2} / 4 \delta^{2}}}{\rho_{n}(0)}
$$

where, by comparison with eq. (7), the coefficients $A_{n}$ and $B_{n}$ are given by

$$
\begin{aligned}
& A_{n}=1+\frac{\langle p(p-1)\rangle_{z}}{\langle p\rangle_{z}}, \\
& B_{n}=\frac{1}{2 \delta \sqrt{ } \pi} \frac{\langle p(p-1)\rangle_{z}}{\langle p\rangle_{z}}
\end{aligned}
$$

One can then determine from the data the ratio $\langle p(p-1)\rangle_{z} /\langle p\rangle_{z}$ for each mult1plicity Since in inclusive correlations, this ratio is only measured at $z=1$, it is clear that semi-inclusive correlations provide some important information on cluster decay

We choose to compare three different cases of cluster decay The first one corresponds to the decay into a fixed number, $p_{0}$, of particles * (for definiteness, we consider the decay into negative particles) This case has already been considered in ref. [1] and corresponds to

$$
\frac{\langle p(p-1)\rangle_{z}}{\langle p\rangle_{z}}=p_{0}-1
$$

* More generally one can take

$$
w(p)=1-a+a \delta\left(p-p_{0}\right),
$$

where $(1-a)$ is the probability that the clusters decay into neutral particles only 
independently of $z$. The second case is that of the $\rho-\rho$ model [13] Each cluster decays into $\left(2 \pi^{+}, 2 \pi^{-}\right)$with a probability $\frac{1}{3}$ and into $\left(\pi^{+}, \pi^{-}, 2 \pi^{0}\right)$ with a probabilty $\frac{2}{3}$ One has

$$
\begin{aligned}
& z=\frac{1}{2}(-1+\sqrt{1+8 n / \bar{n})}, \\
& \frac{\langle p(p-1)\rangle_{z}}{\langle p\rangle_{z}}=\frac{z}{z+1}
\end{aligned}
$$

Finally we consider that $w(p)$ is a Poisson distribution for $p \geqslant 1$ with parameter $p_{0}$, while $w(0)=0^{*}$ The average number of particles is then given by $\langle p\rangle=p_{0}\left(1-\mathrm{e}^{-p_{0}}\right)^{-1}$, and

$$
\frac{\langle p(p-1)\rangle_{z}}{\langle p\rangle_{z}}=p_{0} z,
$$

where $z$ is solution of the equation

$$
p_{0} z \mathrm{e}^{p_{0} z}=\frac{n}{\bar{k}}\left(\mathrm{e}^{p_{0}}-1\right) \text {. }
$$

In order to make a useful comparison we require that all three models give identical inclusive correlations, $1 \mathrm{e},\langle p(p-1)\rangle_{z} /\langle p\rangle_{z}$ is the same at $z=1$ We then have $p_{0}=\frac{3}{2}$ (mathematical example only') in (9) and $p_{0}=\frac{1}{2}(\langle p\rangle=127)$ in (11).

In table 1 we give the results of this comparison with the following values of the parameters

$$
\bar{k}=\frac{15}{4}, \quad Y=6,
$$

corresponding to the top ISR energy, with an average negative multiplicity of 5 It can be seen from table 1 that the ratio $\langle p(p-1)\rangle_{z} /\langle p\rangle_{z}$, which is constant in the first case, increases with $n$ in the $\rho \rho$ and Poisson models. This is in fact a general property of cluster models using the fact that $\lambda(z)$ is a polynomial in $z$ with positive coefficients, it is easy to show that $\langle p(p-1)\rangle_{z} /\langle p\rangle_{z}$ is always an increasing function of $z$, and thus of $n$.

Table 1 illustrates the importance of extracting from the data the ratio $\langle p(p-1)\rangle_{z} /\langle p\rangle_{z}$, since it appears that this quantity is rather sensitive to the properties of cluster decay, whereas $R_{n}(0,0)$ is much less so, because of cancellations arising from the presence of the $1 / n$ term in eq (7)

We now consider the experimental data which are avallable from a $205 \mathrm{GeV} \mathrm{NAL}$ bubble chamber experiment [6], and also from the Pisa-Stony Brook experiment [7] at $\sqrt{ } s=23$ and $62 \mathrm{GeV}$. The lower energy ISR and the NAL data, which are at the same center-of-mass energy, are inconsistent with each other. We have chosen to

\footnotetext{
* $w(0)$ is the probability for the cluster to decay into neutral particles only Hence our choice of $w(0)$ is somewhat arbitrary
} 
Table 1

$\langle p(p-1)\rangle\left\langle\langle p\rangle\right.$ and $R_{n}^{\mathrm{cc}}(0,0)$ as a function of $n_{\mathrm{c}}$ for the three cluster decay models under consideration

\begin{tabular}{|c|c|c|c|c|c|c|}
\hline & $n_{\mathrm{c}}$ & 8 & 12 & 16 & 20 & 24 \\
\hline $\begin{array}{l}w(p)= \\
\delta\left(p-p_{0}\right)\end{array}$ & $R_{n}^{\mathrm{cc}}$ & 031 & 018 & 013 & 010 & 008 \\
\hline \multirow{2}{*}{$\rho \rho$ model } & $\frac{\langle p(p-1)\rangle}{\langle p\rangle}$ & 184 & 2 & 212 & 218 & 224 \\
\hline & $R_{n}^{\mathrm{cc}}$ & 027 & 018 & 014 & 012 & 010 \\
\hline \multirow{2}{*}{$\begin{array}{l}\text { Poisson } \\
\text { dist }\end{array}$} & $\frac{\langle p(p-1)\rangle}{\langle p\rangle}$ & 170 & 2 & 224 & 236 & 262 \\
\hline & $R_{n}^{\mathrm{cc}}$ & 024 & 018 & 016 & 013 & 012 \\
\hline
\end{tabular}

In the case $w(p)=\delta\left(p-p_{0}\right)$ the ratio $\langle p(p-1)\rangle /\langle p\rangle=2$ independently of $n_{\mathrm{c}}$ The value of $n$ chosen to evaluate $R_{n}$ from eq (7) was $n=n_{\mathrm{c}}-2$ The cholce of the parameters $\bar{k}$ and $Y$ corresponds to the top ISR energy Our comparison is made here for charged particles, in order to compare our values for $R_{n}^{\mathrm{cc}}$ with the ISR results

compare the models with the NAL results, which are given in terms of rapidity for each well defined multıplicity.

The parameter $Y$ is evaluated using the equation

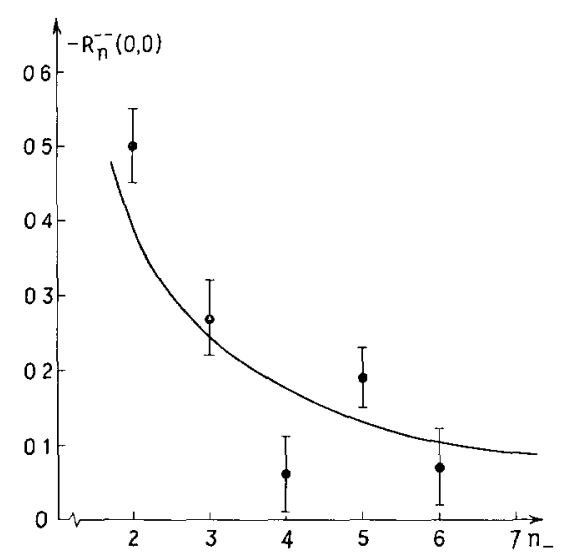

Fig 1 A plot of $-R_{n}^{--}(0,0)$ versus $n$, , where $R_{n}^{--}(0,0)$ is the semi-inclusive correlation function for two negatively charged particles with zero rapidities We compare the $205 \mathrm{GeV}$ NAL data with the following models for cluster decay (1) fixed number of negative pions, (11) $\rho-\rho$ model, (iii) Poisson distribution (see sect 3 for more details) As the three models give practically identical results, we have drawn only one curve 


$$
Y=\frac{\bar{n}}{(1 / \sigma) \mathrm{d} \sigma / \mathrm{d} y} \approx 3.85,
$$

while $\bar{k}=\frac{3}{4} \bar{n}_{-}$1s determined using the "non-diffractive" value [14] $\bar{n}_{-}=3.3$ of $\bar{n}_{-}$ It is seen from fig 1 that $R_{n}^{--}(0,0)$ is satisfactorily described by the models We have also calculated the correlation for charged particles $R_{n}^{\mathrm{cc}}(0,0)$ and find that it is essentially zero for all $n$ (more precisely $R_{n}^{\mathrm{cc}}(0,0)$ lies between 0 and 002 ), in agreement with the data. The limited statistics and the small length of the rapidity plot do not allow to make an analysis of the $y$ dependence

It turns out that the models also give a satisfactory description of the $\sqrt{ } s=62$ GeV Pisa-Stony Brook data (see table 1), but the disagreement of the NAL and ISR results at $\sqrt{ } s=23 \mathrm{GeV}$ must be explained before one can draw any conclusions However, a complete analysis at the top ISR energy would be extremely useful since one may hope to separate the two terms in $R_{n}$ (eq. (7))

\section{Correlations between charged and neutral particles}

It would be interesting to have some information, not only on the distribution of charged particles, but also on the distribution of neutral particles within a cluster To analyze this problem, it is useful to introduce the generating function $\Lambda\left(z, z_{0}\right)$ for cluster decay into negative and neutral pions (9)

$$
\Lambda\left(z, z_{0}\right)=\sum_{p, p_{0}} z^{p} z^{p_{0}} w\left(p, p_{0}\right)
$$

In eq $(12), w\left(p, p_{0}\right)$ is the probability for a cluster to decay into $p$ negative and $p_{0}$ neutral pions. We will also need the derivatives of $\Lambda\left(z, z_{0}\right)$.

$$
\Lambda^{(m, n)}\left(z, z_{0}\right)=\frac{\partial^{m+n}}{\partial z^{m} \partial z_{0}^{n}} \Lambda\left(z, z_{0}\right),
$$

Notice finally that $\lambda(z)=\Lambda(z, 1)$ We will first show that in the case of independent cluster production, the average number $\overline{n_{0}\left(n_{-}\right)}$of $\pi^{0}$ 's as a function of $n_{-}$must be an increasing function of $n_{-}$. The general formula for $\overline{n_{0}\left(n_{-}\right)}$is

$$
\overline{n_{0}\left(n_{-}\right)}=\bar{k} \Lambda^{(0,1)}(z, 1) \text {, }
$$

where $z$ is again given by eq (5a) Since $\Lambda\left(z, z_{0}\right)$, is a polynomial in $z$ and $z_{0}$ with positive coefficients, $z$ must be an increasing function of $n_{-}$, and $\overline{n_{0}\left(n_{-}\right)}$must be an increasing function of $z$ In the case of the $\rho-\rho$ model one obtains

$$
\overline{n_{0}\left(n_{-}\right)}=\frac{4}{3} \bar{k} z=\frac{2}{3} \bar{k}\left(-1+\sqrt{1+8 n_{-} \sqrt{n_{-}}}\right)
$$

This expression gives an excellent fit of $\overline{n_{0}\left(n_{-}\right)}$for $n_{-} \leqslant 6$ at $205 \mathrm{GeV}$ [15] (fig 2) but falls to reproduce the decrease of $\overline{n_{0}\left(n_{-}\right)}$when $n_{-}$is larger than 6 We believe that this is an indication that for neutral clusters the cluster multiplicity distribution is not Poisson, as will be discussed in more detall in the next section 


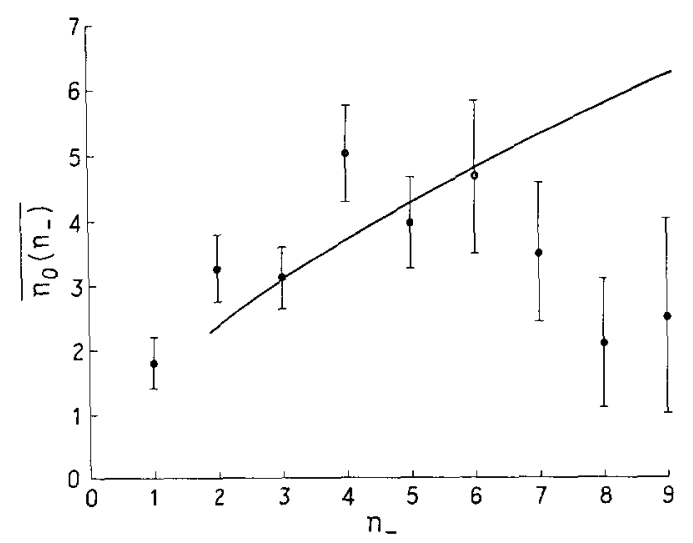

Fig 2 Comparison of the $\rho-\rho$ model with the $205 \mathrm{GeV}$ data on $\overline{n_{0}\left(n_{-}\right)}$, the average number of $\pi^{0}$ 's produced for a given number of $\pi^{-} \mathrm{s}$, in the case of independent clusters

We would also like to suggest that the $n$-dependence of the rapidity correlations between a negative (or charged) particle and a $\pi^{0}$ (in practice a $\gamma$-ray) at a fixed number of prongs can give some useful information on the distribution of neutral particles in a cluster Indeed, one can easily prove the following equation

$$
\frac{1}{n_{-} \sigma_{n_{-}}} \frac{\mathrm{d}^{2} \sigma_{n_{-}}}{\mathrm{d} y_{-} \mathrm{d} y_{0}}=\frac{\overline{n_{0}\left(n_{-}\right)}}{Y^{2}}+\frac{\Lambda^{(1,1)}(z, 1)}{\lambda^{\prime}(z)}\left(P^{(2)}\left(y_{-}, y_{0}\right)-\frac{1}{Y^{2}}\right) \text {, }
$$

where $y_{-}$and $y_{0}$ are the rapidity of the negative and neutral particles respectively The ratio

$$
\Lambda^{(1,1)}(z, 1) / \lambda^{\prime}(z)
$$

is to be interpreted as the $z$-dependent average of $p p_{0}$ divided by $\langle p\rangle$

$$
\frac{\Lambda^{(1,1)}(z, 1)}{\lambda^{\prime}(z)}=\frac{\left\langle p p_{0}\right\rangle_{z}}{\langle p\rangle_{z}}
$$

The inclusive correlation between negative and neutral pions measures this ratio at $z=1$, but the semi-inclusive correlations (16) allow to study its variation with $z$

In order to illustrate eq. (16), we briefly discuss 3 examples

(1) No correlations between negative and neutral pions within a cluster Then

$$
\left\langle p p_{0}\right\rangle_{z} /\langle p\rangle_{z}=\left\langle p_{0}\right\rangle
$$

independently of $z$

(11) The cluster decays either into charged particles only, or into neutral particles only Then,

$$
\left\langle p p_{0}\right\rangle_{z} /\langle p\rangle_{z}=0
$$


(111) The $\rho-\rho$ model. Here

$\left\langle p p_{0}\right\rangle_{z} /\langle p\rangle_{z}=\frac{2}{z+1}$.

\section{Production of non-independent clusters}

If the clusters are not independent, 1 e., if their multiplicity distribution $Q(k)$ does not follow a Poisson law, it is still possible to derive eq. (7) for $R_{n}$. Although, superficially, it seems that only quantities related to cluster decay appear in $R_{n}$, this is not true since the relation between $n$ and $z$ [(eq. (5a)] depends on the shape of $Q(k)$, and therefore on the cluster production mechanism.

Whereas for independent cluster emission only the average number of clusters, a quantity independent of $z$, appears in eq (5a), such is not the case for non-independent clusters. It then becomes difficult to disentangle the contributions of production and decay mechanisms to semi-inclusive correlations

However, we believe that it is still useful to determine experimentally the ratio

$$
r(n)=\frac{\langle p(p-1)\rangle_{n}}{\langle p\rangle_{n}},
$$

by measuring the coefficients $A_{n}$ and $B_{n}$ in relation (8), provided the multiplicity distribution $P(n)$ is avalable. Indeed, if we consider some precise form for $w(p)$ and assume we know $P(n)$, it should be possible, at least in principle, to calculate the cluster multiplicity distribution $Q(k)$ and then $r(n)$. Therefore, one ought to be able to express $r(n)$ directly as a function of $P(n)$ and to check whether the predicted form agrees with the experimental data on $r(n)$.

Let now $G(\lambda)$ be the generating function for cluster production

$$
G(\lambda)=\sum \lambda^{k} Q(k)
$$

$P(n)$ is then given by

$$
\begin{aligned}
P(n) & =\left.\frac{1}{n^{\prime}} \frac{\partial^{n}}{\partial z^{n}} G(\lambda(z))\right|_{z=0} \\
& =\left.\frac{1}{n^{\prime}} \frac{\partial^{n-1}}{\partial z^{n-1}} \lambda^{\prime}(z) G^{\prime}(\lambda(z))\right|_{z=0}
\end{aligned}
$$

On the other hand, it is easily shown, using for example, the formalism of ref [1], that

$$
P_{1}(n) \equiv\langle p(p-1)\rangle_{n}=\left.\frac{1}{n^{\prime}} \frac{\partial^{n}}{\partial z^{n}} z^{2} \lambda^{\prime \prime}(z) G^{\prime}(\lambda(z))\right|_{z=0},
$$




$$
\langle p\rangle_{n}=\left.\frac{1}{n^{\prime}} \frac{\partial^{n}}{\partial z^{n}} z \lambda^{\prime}(z) G^{\prime}(\lambda(z))\right|_{z=0}=n P(n)
$$

Then, replacing $\lambda(z)$ by its expression in terms of $w(p), P(n)$ and $P_{1}(n)$ can be rewritten as

$$
\begin{aligned}
& P(n)=\left.\sum_{p=1}^{n} \frac{p w(p)}{n(n-p)^{\prime}} \frac{\partial^{n-p}}{\partial z^{n-p}} G^{\prime}(\lambda(z))\right|_{z=0}, \\
& P_{1}(n)=\left.\sum_{p=2}^{n} \frac{p(p-1) w(p)}{(n-p)^{1}} \frac{\partial^{n-p}}{\partial z^{n-p}} G^{\prime}(\lambda(z))\right|_{z=0}
\end{aligned}
$$

Let us now study two relevant examples.

(1) Each cluster decays into either one or two negative particles, as is the case for the $\rho \rho$ model

Here $\lambda(z)=z w(1)+z^{2} w(2)$, and $P_{1}(n)$ is determined by the recursion formula

$$
P_{1}(n)=\frac{2 w(2)}{w(1)}\left((n-1) P(n-1)-P_{1}(n-1)\right)
$$

In the $\rho \rho$ model $w(1)=\frac{2}{3}$ and $w(2)=\frac{1}{3}$

(11) The cluster decay is a truncated Poisson distribution, $1 \mathrm{e}, w(0)=0$ Then $\lambda(z)=\left(1-\mathrm{e}^{-p_{0}}\right)^{-1}\left(\mathrm{e}^{z p_{0}}-1\right)$ and one finds immediately from relations (17) and (18) that.

$$
P_{1}(n)=p_{0}(n-1) P(n-1)
$$

and consequently

$$
r(n)=\frac{\langle p(p-1)\rangle_{n}}{\langle p\rangle_{n}}=p_{0} \frac{n-1}{n} \frac{P(n-1)}{P(n)}
$$

Th1s formula was first derived by Berger [16]

It is thus clear that the experimental determination of the ratio $\langle p(p-1)\rangle_{n} /\langle p\rangle_{n}$ is an important tool in investigating cluster models While its magnitude relates to the properties of the cluster decay distribution, its variation with $n$ gives an important clue as to what extent clusters are produced independently. This variation is strongest when clusters are non-1ndependent This point is demonstrated in figs $3 a, b$ where in each case, fig. $3 a$ for the $\rho \rho$ model, $f_{1} g$ $3 b$ for the truncated Poisson model, we compare the $r(n)$ obtained on one hand in an independent cluster model, as done in sect. 3, and on the other hand, derived directly from the topological cross sections To do the latter calculation, we assume a Poisson distribution for negative particles, in agreement with experımental data at $205 \mathrm{GeV}$ on the non-diffractive component [14], and use the expressions given above in (1) and (11)

The previous remarks can also be understood looking at $f_{2}$, the second Mueller moment At $205 \mathrm{GeV}, f_{2}^{-}=0$ for the non-diffractive component [14]. This implies that $f_{2}^{\text {cluster }}$ is negative since by integration of the inclusive two-particle correlation we have 


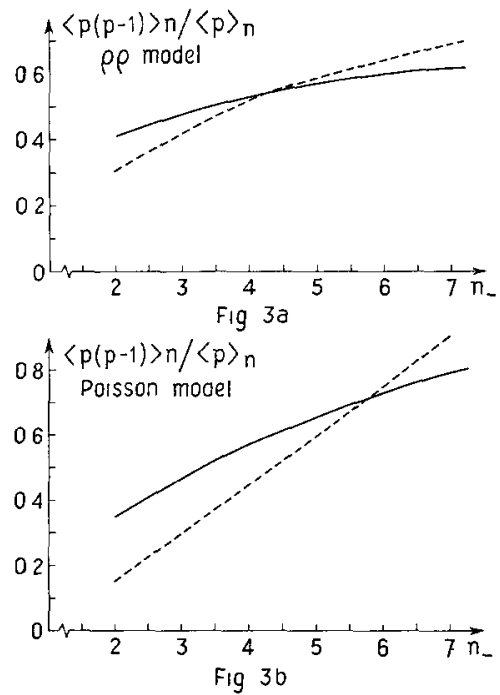

Fig 3 The ratio $\langle p(p-1)\rangle\langle\langle p\rangle$ as a function of $n$ (a) The $\rho-\rho$ model (b) The Poisson model for cluster decay The full lines correspond to independent cluster emission, the dashed lines to a Poisson distribution for negative particles

$$
f_{2}=f_{2}^{\text {cluster }}\langle p\rangle^{2}+\bar{k}\langle p(p-1)\rangle \text {. }
$$

We have checked that a negative value for $f_{2}^{\text {cluster }}$ leads to an increase with $n$ of $r(n)$ which is faster than in the case of independent clusters, except of course, when the number of charged particles in a cluster is fixed Therefore, in general, in cluster models $R_{n}$ is expected to fall off with $n$ more slowly than $1 / n$

\section{Summary}

We here summarize the main points made in the preceeding sections. We first established a general formula (eq. (4)), valid in all short-range order models, that expresses the semi-inclusive correlation function in terms of the inclusive one We then gave the specific form (eq (7)) this formula takes for $R_{n}(0,0)$ in the case of neutral cluster models The expression for $R_{n}(0,0)$ depends essentially on the ratio $\langle p(p-1)\rangle_{z} /\langle p\rangle_{z}$, which is characteristic of cluster properties A sensitive test of cluster models therefore consists in extracting from the data this quantity However, though eq. (7) is true in all cluster models, it is useful only when clusters are produced independently, in which case the relation between the variable $z$ and the number $n$ of particles is simple. When clusters are not independent, it is advantageous to relate $\langle p(p-1)\rangle_{n} /\langle p\rangle_{n}$, directly to the topological cross sections. Thus, in all cases, the determination of the ratio $\langle p(p-1)\rangle_{n} /\langle p\rangle_{n}$, in particular its variation with $n$, is crucial if one wants to learn about clusters, their decay mechanism and their production 
We wish to thank Ed Berger for stımulatıng discussions

\section{References}

[1] E L Berger, CERN preprint (1974) TH 1816

[2] J Ranft and G Ranft, Phys Letters 49B (1974) 286

[3] A Biatas, Invited talk at the 4th Conf on hadrodynamics, Pavia, CERN preprint (1973) TH 1745

[4] R C. Arnold and G H Thomas, ANL preprint (1974) HEP 7401

[5] L Canesch1, CERN preprint (1974) TH 1826

[6] R Singer et al, ANL preprint (1974) HEP 7369

[7] G Bellettinı, Invited talk at the Stony Brook Conf AIP Conf Proc, ed C Quigg (1973)

[8] W Frazer, R Pecce1, S Pinsky and C I Tan, Phys Rev D7 (1973) 2647

[9] M Le Bellac, Acta Phys Pol B4 (1973) 901

[10] P Pirla and S Pokorski, Phys Letters 43B (1973) 502,

F Hayot and A Morel, Saclay preprint (June 1973), Vucl Phys B68 (1974) 323

[11] A Biatas, K Fialkowski and K Zalewsk1, Phys Letters 45B (1973) 337

[12] L Foá, Invited talk at the 9th Monond meeting (1974)

[13] F Hayot and A. Morel, Nuovo Cimento Letters 7 (1973) 221, 8 (1973) 71

[14] R Singer et al, ANL preprint HEP 7367 (1973)

[15] K Jaeger et al, Results presented at the AIP Meeting on particles and fields, AIP Conf Proc, ed H Bingham (1973).

[16] E L Berger, private communication 